
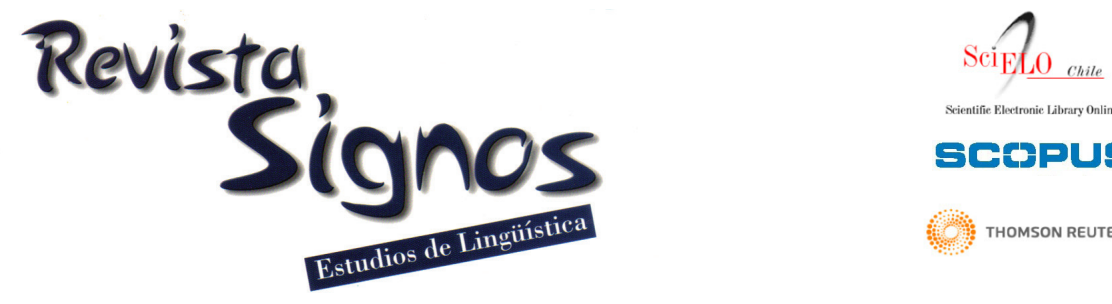

\title{
Apoyos co(n)textuales en el empleo discursivo de las unidades fraseológicas'
}

\section{Co(n)textual supports in the discursive use of phraseological units}

\author{
Inés Olza \\ iolzamor@unav.es \\ Universidad de Navarra \\ España
}

\author{
$M^{a}$ Carmen Losada \\ mcarmen.losada@usc.es \\ Universidad de Santiago de Compostela \\ España
}

Recibido: 27-IV-2010 / Aceptado: I5-XI-20I0

Resumen: El objetivo de este artículo es definir y caracterizar la tendencia -observada en el empleo discursivo de las unidades fraseológicas (UFS)- por la que los fraseologismos suelen coordinarse en su co(n)texto cercano con otras unidades léxicas o fraseológicas que orientan y especifican su interpretación, y que etiquetamos aquí, por ello, como 'apoyos co(n)textuales'. El trabajo examina, en primer lugar, esta tendencia en el marco de las disciplinas -lingüística cognitiva y psicolingüística, por un lado, y lingüística y semántica textuales, por otro- que han analizado cómo el co(n)texto lingüístico orienta el uso y la interpretación de las expresiones fijas. En segundo lugar, con base en los datos recabados para tres locuciones del corpus FRASESPAL ('coger el toro por los cuernos','no tener pelos en la lengua/sin pelos en la lengua ' $y$ 'tener/quedar a alguien mucha vida por delante'), se abren vías de análisis de la relevancia cuantitativa que la aparición de los apoyos co(n)textuales posee en el empleo discursivo de las UFS. Se presenta, por último, una tipología de las piezas que se ha detectado suelen funcionar con más frecuencia como apoyos co(n)textuales: expresiones que parafrasean el significado fraseológico de partida; expresiones que focalizan un sema específico de dicho significado; unidades léxicas y fraseológicas sinónimas a la expresión 'central' con la que coaparecen; y unidades léxicas y fraseológicas antónimas a la UF 'central'. La tipología se apoya en testimonios del corpus FRASESPAL obtenidos, principalmente, del Corpus de Referencia del Español Actual (CREA), con apoyo de algunas muestras extraídas de Internet.

Palabras Clave: Fraseología, discurso, significado figurado, lingüística cognitiva, lingüística del texto. 


\begin{abstract}
The aim of this article is to define and describe the tendency -that is observed in the discursive use of phraseological units- by which these units are frequently coordinated in their near co(n)text with other lexical and phraseological expressions that orient and specify their interpretation and can be, thus, labelled as 'co(n)textual supports'. The paper firstly examines this tendency in the frame of the disciplines-on the one hand, cognitive linguistics and psycholinguistics, and, on the other hand, text linguistics and text semantics - that have analyzed how the linguistic co(n)text orients the use and interpretation of fixed expressions. In the second place, on the basis of the data obtained for three expressions of the FRASESPAL corpus (coger el toro por los cuernos, no tener pelos en la lengua/sin pelos en la lengua and tener/quedar a alguien mucha vida por delante), we open some ways of analysis of the quantitative relevance of the occurrence of these co(n)textual supports in the discursive use of idioms. Finally, we present a typology of the units that function more often as co(n)textual supports: expressions that paraphrase the initial phraseological meaning; expressions that highlight a specific seme of this meaning; lexical and phraseological units that are synonymous with the 'central' expression they co-occur with; and lexical and phraseological units that are antonymous with the 'central' expression. The typology is based on the FRASESPAL data, which are drawn mainly from the Corpus de Referencia del Español Actual (CREA) and also-though secondarily-from the Internet.
\end{abstract}

Key Words: Phraseology, discourse, figurative meaning, cognitive linguistics, text linguistics.

\title{
INTRODUCCIÓN
}

En este trabajo se propone ahondar en lo que se ha observado como una 'tendencia significativa' en el 'empleo discursivo real' de las unidades fraseológicas (UFS):aquella por la que, en su inserción particular en el discurso, los fraseologismos suelen ir acompañados en su contexto o, más bien, en su 'cotexto' lingüístico cercano por otras expresiones que 'apoyan', 'orientan' y 'especifican' su interpretación. Con el objetivo de definir el alcance y las razones de aparición de estos apoyos co(n)textuales, el artículo se estructura en cuatro secciones: en primer lugar, en el apartado I. se define de manera general esta tendencia discursiva; en la sección 2. se explora, desde una perspectiva más amplia, el papel que desempeñan el 'contexto cognitivo' y el 'cotexto lingüístico' como soportes orientadores del empleo discursivo de las expresiones fraseológicas; en el apartado 3. se ofrecen algunos datos basados en el análisis del corpus FRASESPAL' que apuntan hacia la 'relevancia cuantitativa' que posee la aparición de los apoyos co(n)textuales en los usos fraseológicos reales y que permiten caracterizar con más precisión su funcionamiento; $y$, finalmente, en la sección 4 . se propone una 'tipología' -no exhaustiva, aunque sí representativa- de los elementos y mecanismos que suelen actuar como apoyos co(n)textuales en el uso discursivo de las UFS.

Este estudio se encamina, por tanto, a nutrir el análisis de la fraseología 'en contexto'; análisis que reclama, sin duda, cada vez más esfuerzos que completen la aproximación a las UFS desde disciplinas como la Pragmática o el Análisis del Discurso. En efecto, si bien la perspectiva pragmática parece ya consolidada en los estudios fraseológicos del español -fundamentalmente, en la caracterización de los tipos de UFS de valor pragmático-discursivo ('fórmulas rutinarias' y 'locuciones marcadoras', principalmente ${ }^{2}$-, no abundan los trabajos que analicen monográficamente el 'comportamiento discursivo real' no solo de las unidades de valor pragmático, sino también de las que se pueden definir como meramente descriptivas. Unas y otras tendrán, por tanto, cabida en este trabajo.

\section{Empleo de los apoyos co(n)textuales de las UFS: Definición preliminar de una tendencia discursiva}

Según se ha señalado en la introducción, lo que aquí se etiqueta como 'apoyos co(n)textuales' no pretende definirse como una (nueva) categoría funcional, sino más bien como la evidencia lingüística de una 'tendencia' que, según se explicará en el apartado 2. (especialmente en la sección 2. I.), posee enraizamientos cognitivos, y por la que las UFS establecen lazos semántico-discursivos con otras secuencias léxicas y fraseológicas de su cotexto lingüístico que apoyan de modos diversos su interpretación idiomática. Este es el tipo de vínculo que se aprecia en los ejemplos (I) y (2) entre las UFS 'coger el toro por los cuernos' y '[ser] (un) hombre nuevo' y sus respectivos apoyos ('afrontar de manera decidida los problemas' y 'nacer de nuevo'): 
(I) Asimismo, añadió que "la Generalidad ha cogido el toro por los cuernos para afrontar de manera decidida los problemas provocados por las fuertes lluvias" (ABC (España), 1997, CREA).

(2) De la noche a la mañana mi papá cambió. Dejó de tomar licor. Un día nos dijo que ya era un hombre nuevo, que había nacido de nuevo y nos pidió que fuéramos con él a su iglesia (La Hora (España), 1997, CREA).

Dicho de otro modo, no estamos tratando con una categoría de piezas de rasgos (formales, sintácticos o pragmáticos) homogéneos, sino más bien con los 'puntales' visibles de un movimiento cognitivo-discursivo activado -como se ilustrará más claramente en las secciones 2. y 4.- por las peculiaridades semánticas y estilísticas que suelen poseer las UFS ${ }^{3}$; movimiento para el que, en principio, cualquier secuencia léxica y fraseológica puede llegar a funcionar como 'puntal'. Dicho movimiento se puede caracterizar ahora generalmente como la necesidad de que el significado de la UF se glose o especifique de modos diversos, dado el carácter figurativo y estilísticamente marcado de los fraseologismos y también, en ocasiones, dada la vaguedad semántica asociada a las expresiones fraseológicas. En los apartados 2. y 3. se definen e ilustran con más precisión la razones de aparición de los apoyos co(n)textuales de las UFS.

\section{Papel general del co(n)texto en el uso y la interpretación de los fraseologismos}

Se incluyen aquí algunas reflexiones sobre los modos en el que el co(n)texto de empleo de las UFS -y los apoyos co(n)textuales dentro de élfavorece y activa su aparición, y contribuye también en no pocas ocasiones a orientar su interpretación idiomática. La red de relaciones que se establece entre los fraseologismos y su co(n)texto de empleo se presentará, primeramente, desde la perspectiva de la lingüística cognitiva y la psicolingüística (sección 2. I.); y, en segundo lugar, desde la óptica de la lingüística y la semántica textuales (apartado 2.2.).

\section{I. Perspectiva de la lingüística cognitiva y la psicolingüística}

Desde una óptica 'cognitiva' y 'psicolingüística', puede tenerse en cuenta, en primer lugar, cómo ciertos contextos figurativos suelen propiciar la aparición de UFS que sigan ilustrando los mismos esquemas metafóricos y metonímicos sobre los que se asientan. Dicho de otro modo, la transparencia figurativa que presentan de por sí ciertas expresiones idiomáticas se suele ver favorecida por su inserción en contextos que se orientan conceptualmente hacia el mismo patrón figurativo que las genera (Gibbs, 1994; Olza, 2009). Esto es lo que se aprecia, por ejemplo, en el siguiente pasaje, extraído de un experimento comentado por Gibbs (1993, 1994), donde las expresiones subrayadas (very tense, making her fume, getting hotter, blew her top) ilustran el esquema metafórico LA IRA ES UN LIQQUIDO CALIENTE DENTRO DE UN CONTENEDOR y preparan el marco cognitivo adecuado para la aparición de la locución metafórica to blow one's top/ stack ('reventársele a alguien la chimenea' -> 'estallar de ira'):

Mary was very tense about the evening's dinner party. The fact that Bob had not come home to help was making her fume. She was getting hotter with every passing minute. Dinner would not be ready before the guests arrived. [...] When Bob strolled at ten minutes to five whistling and smiling, Mary blew her top/stack.

Mary estaba muy tensa y nerviosa con la cena de aquella noche. Echaba humo porque Bob no había vuelto a casa para ayudar. Mary se iba calentando cada vez más, minuto a minuto. La cena no estaría lista antes de que llegaran los invitados. [...] Cuando Bob apareció tranquilamente a las cinco menos diez silbando y sonriendo, Mary estalló de ira. (Gibbs, 1993: 70. La traducción al español es nuestra).

El co(n)texto citado es, por tanto, propicio al empleo de fraseologismos que continúen ilustrando esa misma metáfora conceptual, y no a la aparición de otro tipo de expresiones que, aunque también denotan 'enfado', se basan en otros esquemas de correspondencias (por ejemplo, to bite one's head off, 'morder la cabeza de alguien' -> 'mostrar furia contra alguien', que se sustenta en otra metáfora distinta: LA IRA ES UN COMPORTAMIENTO ANIMAL) (Gibbs, I 993, 1994). Como cabía esperar, las respuestas de los sujetos del experimento -a quienes se les presentaron distintas opciones (to blow one's top/stack, to bite one's head off y otras) para cerrar el texto- apuntaron en este mismo sentido, lo que podría tomarse como un indicio más de que los hablantes comprenden las diferencias entre las correspondencias figurativas que motivan las UFS 'en estrecha relación con su co(n)texto de empleo'. 
Aplicando estas observaciones al examen de los ejemplos (I) y (2), reproducidos en el apartado I., se aprecia que la expresión de las nociones de 'asumir problemas' (I) y 'experimentar un cambio sustancial' (2) se articula con base en los esquemas metafóricos ASUMIR PROBLEMAS ES CONFRONTARSE (FÍSICAMENTE) CON ELLOS y EXPERIMENTAR UN CAMBIO SUSTANCIAL ES VOLVER A NACER, respectivamente, sobre los que inciden de modo conjunto y figurativamente coherente las UFS 'coger el toro por los cuernos' y '[ser] (un) hombre nuevo', y sus apoyos 'afrontar de manera decidida los problemas' y 'nacer de nuevo'. El empleo coordinado de las citadas UFS y sus respectivos apoyos co(n) textuales responde, pues, como se explicará también en la sección 3. (especialmente en el apartado 3.2.), a la actualización lingüística de un mismo patrón de pensamiento figurativo.

\subsection{Perspectiva de la lingüística y la semántica textuales}

La aparición de los apoyos co(n)textuales, que en la sección 2.I. se ha presentado como ligada a la orientación conceptual y figurativa de los discursos, puede abordarse, igualmente, desde la lingüística y la semántica del texto como una de las manifestaciones lingüísticas de la 'coherencia' textual, como un mecanismo garante de la 'cohesión' textual, y/o, más bien, como un fenómeno enmarcado -en un sentido muy amplio- dentro de la 'isotopía semánticodiscursiva', entendida esta como la redundancia o iteración de semas en la estructuración conceptual e informativa del mensaje (Rastier, 1976).

Volviendo a los testimonios de los ejemplos (I) y (2), puede decirse que la coherencia temática y conceptual de cada pasaje en torno a las nociones 'asumir problemas' y 'experimentar un cambio sustancial' es la que explica, de un lado, la aparición central de las locuciones "coger el toro por los cuernos' y '[ser] (un) hombre nuevo'; y, de otro, su apoyo periférico en expresiones semánticamente paralelas a ella ('afrontar de manera decidida los problemas' y 'nacer de nuevo'). La coordinación en el empleo de las UFS y sus respectivos apoyos co(n)textuales podría entenderse, de hecho, como una forma más de 'recurrencia léxico-semántica' (van Dijk, 1972; Casado, 1993), que contribuye, igualmente, a reforzar las bases de 'cohesión' del texto.

En otro orden de cosas, interesa comparar, asimismo, la tendencia de aparición de los apoyos co(n)textuales con otro fenómeno discursivo que también responde, en cierto modo, al carácter estilística y figurativamente marcado de las UFS. Otros acercamientos, que desde la lingüística textual han ponderado el papel que ciertos segmentos co(n)textuales desempeñan en la interpretación de las UFS, han definido y analizado el comportamiento de los llamados 'marcadores', 'conectores' o 'presentadores fraseológicos' (Corpas Pastor, 1996; Sancho Cremades, 1999; Olímpio de Oliveira Silva, 2006; Mellado Blanco, 2009; Olza, 20I0), que introducen, indicándolo expresamente, una unidad de discurso repetido ${ }^{4}$. Se trata de expresiones de naturaleza (cuasi)fraseológica- como 'como (vulgarmente) se dice', 'como dice el refrán', 'como dicen en mi pueblo' o 'dicho mal y pronto', que sirven para introducir o enmarcar en el discurso cualquier tipo de UF4. Interesa observar aquí la función primordial de 'preparar', orientar y completar la interpretación fraseológica del segmento al que acompañan; función que se suele complementar con otras no menos importantes, como poner de manifiesto, por ejemplo, el carácter diatópica ('como dicen en mi pueblo'), diastrática o diafásicamente ('como vulgarmente se dice', 'dicho mal y pronto') marcado de muchas UFS 5 .

(3) Las responsabilidades políticas hay que asumirlas, con independencia de lo que luego digan los jueces. El vicepresidente cometió el error de no coger el toro por los cuernos, como vulgarmente se dice, y luego tampoco ha sabido actuar con entereza conforme han ido sucediéndose las denuncias sobre las actividades de sus hermanos (Tiempo (España), 1990, CREA).

\section{Marcador fraseológico Apoyos co(n)textuales}

El ejemplo (3) puede servir para relacionar el valor de estos marcadores fraseológicos con el del tipo de elementos que se describen y analizan en este trabajo como apoyos co(n)textuales. En efecto, en este empleo discursivo de la locución 'coger el toro por los cuernos' se identifican varios elementos 'periféricos' que, conjuntamente, especifican y orientan su interpretación como expresión idiomática: de un lado, el marcador fraseológico 'como vulgarmente se dice' explicita la conciencia del hablante de que dicha locución pertenece al discurso repetido y está marcada, además, como perteneciente al registro coloquial; $y$, de otro, los apoyos co(n)textuales 'asumir' $y$ 'actuar con entereza' 'reconstruirían', precisamente, la alternativa formal que 'no se dice vulgarmente', es decir, constituyen 
una paráfrasis -no figurativa y diafásicamente no marcada- de lo expresado por la UF.

Lo que aquí etiquetamos como 'apoyo co(n)textual' actúa, pues, más bien en el nivel del 'significado'6 de los fraseologismos, glosando, completando o especificando su contenido, como se comprobará en los epígrafes que siguen (secciones 3. y 4.), y reforzando también, en consecuencia, la isotopía semántica de la secuencia discursiva en que se inserta. Los mencionados marcadores fraseológicos se relacionan, en cambio, con otras dimensiones del uso de las UFS: actitud enunciativa el hablante; mención de marcas connotativas o de adscripción diatópica, diastrática o diafásica del fraseologismo; etc.

En el marco de estas reflexiones sobre la influencia del co(n)texto lingǘístico-cognitivo en el empleo discursivo de las UFS, y una vez que se ha podido caracterizar de modo general la tendencia de aparición de los apoyos co(n)textuales, en el siguiente epígrafe pasaremos a tratar de mostrar en qué medida esta aparición puede llegar a constituir una tendencia cuantitativamente significativa en el uso real de los fraseologismos (apartado 3.I.). Con base en la batería de nuevos ejemplos aportados en la sección 3.I., en el apartado 3.2. se ofrecerá, igualmente, una caracterización más precisa de las funciones más sobresalientes de los apoyos $\operatorname{co}(\mathrm{n})$ textuales.

\section{Una tendencia significativa en el empleo discursivo de las UFS}

\section{I. Indicios cuantitativos: Análisis de una muestra del corpus FRASESPAL}

En el empeño de ilustrar la 'frecuencia relevante' de aparición de los apoyos co(n)textuales, aportaremos aquí algunos datos obtenidos del análisis del corpus FRASESPAL. Los objetivos del Proyecto de Investigación en que trabaja el grupo FRASESPAL se cifran en la construcción de un tesauro fraseológico contrastivo alemán-español, ordenado según criterios onomasiológicos en torno a varios campos conceptuales: principalmente, HABLAR/CALLAR (REDEN/SCHWEIGEN); SALUD/ENFERMEDAD (GESUNDHEIT/KRANKHEIT); (ETAPAS DE LA) VIDA/MUERTE (LEBENSETAPPEN/TOD). El tesauro con el que actualmente se trabaja consta de alrededor de 1600 parejas contrastivas, para que las que se ofrecen, entre otros datos e informaciones, testimonios del uso actual de cada fraseologismo. Pues bien, del manejo de este corpus de UFS y de sus respectivos testimonios surge preliminarmente la observación de la tendencia de empleo de lo que aquí denominamos 'apoyos co(n)textuales' de las UFS. La confirmación de esta tendencia como una constante discursiva cuantitativamente relevante vendrá de la mano de estudios futuros -del corpus FRASESPAL y de otros- de base estadística sistemática. Por el momento, en aras de sustentar provisionalmente la defensa de esta relevancia cuantitativa, ofrecemos una primera cala en esta dirección, centrada en el análisis de los testimonios ofrecidos por el CREA ${ }^{7}$ para tres locuciones del español del corpus FRASESPAL: 'agarrar/coger/ entrar/tomar el/al toro por los cuernos' ('asumir de manera decidida e inmediata un asunto difícil'; DFDEA, 'toro'); 'no tener pelos en la lengua/sin pelos en la lengua' ('decir sin miramientos lo que se piensa'; DFDEA, 'pelo'); 'tener/quedar' a alguien '(toda una/la) vida por delante' ('ser joven $y$, por ende, tener proyectos de vida').

Si lo que se persigue es justificar cuantitativamente la relevancia de coaparición de los apoyos co(n) textuales con estas UFS, un análisis de los testimonios ofrecidos por el CREA en una búsqueda 'bruta' de las citadas expresiones ${ }^{8}$ podrá determinar si en un porcentaje considerable de estos se hallan dichos elementos de apoyo interpretativo. Esto es lo que ocurre, en efecto, en el examen de los citados testimonios, entre los que se registran las siguientes proporciones de aparición de apoyos co(n)textuales.

Según se aprecia en la Tabla I, en nada menos que en un $56,4 \%$ de la muestra de 248 testimonios recabados puede detectarse la presencia de elementos que orientan y especifican la interpretación idiomática de las citadas locuciones. En las secciones 3.2. y 4. citaremos varios de los testimonios de la muestra que poseen apoyos co(n)textuales. A la luz de esta primera tabla, aunque sin intención de polarizar estos resultados, proponemos, por tanto, abrir en el futuro vías más profundas y sistemáticas de análisis de la frecuencia con que el empleo de las UFS se apoya en otras secuencias léxicas y fraseológicas de su co(n)texto que inciden directamente sobre su interpretación. 
Tabla I. Proporciones de aparición de apoyos co(n)textuales en una muestra de testimonios de UFS del corpus FRASESPAL.

\begin{tabular}{|l|l|c|c|c|}
\hline \multicolumn{1}{|c|}{ UF } & $\begin{array}{c}\text { Binomio conceptual } \\
\text { FRASESPAL }\end{array}$ & $\begin{array}{c}\text { Testimonios } \\
\text { CREA }\end{array}$ & $\begin{array}{c}\text { Testimonios } \\
\text { con apoyos } \\
\text { co(n)textuales }\end{array}$ & Proporción \\
\hline $\begin{array}{l}\text { agarrar/coger/entrar/tomar el/al } \\
\text { toro por los cuernos }\end{array}$ & HABLAR/CALLAR & 75 & 35 & $46,6 \%$ \\
\hline $\begin{array}{l}\text { no tener pelos en la lengual } \\
\text { sin pelos en la lengua }\end{array}$ & HABLAR/CALLAR & 92 & 59 & $64 \%$ \\
\hline $\begin{array}{l}\text { tener/quedar a alguien (toda unal } \\
\text { la) vida por delante }\end{array}$ & VIDA/MUERTE & 81 & 46 & $56,8 \%$ \\
\hline \multicolumn{2}{|r|}{ TOTALES } & $\mathbf{2 4 8}$ & $\mathbf{1 4 0}$ & $\mathbf{5 6 , 4 \%}$ \\
\hline
\end{tabular}

\subsection{Observaciones adicionales sobre el funcionamiento de los apoyos co(n) textuales}

Con el objetivo de ilustrar con más precisión el funcionamiento y algunas razones sobresalientes de la aparición de los apoyos co(n)textuales, se partirá aquí del examen de 10 ejemplos representativos de la muestra de la sección 3.I. Con ello se pretende comprender también -según se señaló ya en el apartado I.- en qué medida el empleo de los apoyos está ligado o se halla condicionado por las peculiaridades semánticas y estilísticas de las propias UFS.

(4) Javier le confirmó que estábamos casados con todas las de la ley y le aseguró que él también había tratado de disuadirme, pero en vano. El tío Lucho sugería que volviéramos a Lima cuanto antes, para coger al toro por los cuernos y tratar de arreglar las cosas (Mario Vargas Llosa, La tía Julia y el escribidor (Perú), I 977, CREA).

(5) Llega un momento en los asuntos de los hombres en que hay que coger el toro por los cuernos y enfrentarse a la situación (El Siglo (Panamá), 1997, CREA).

(6) La muerte no es el descanso eterno, no creas esas patrañas. De ella te he hablado en más de una ocasión, con la sinceridad del que no tiene pelos en la lengua. No reposa el que muere, por mucho que no sea creyente, que no sé si es tu caso (Luis Mateo Díez Rodríguez, El oscurecer (Un encuentro) (España), 2002, CREA).

(7) Pues algo de raro tenía mi Esperanza cuando alguien quería tratarle sus amores escondidos de aquellos años. Para lo demás era abierta, franca, sin pelos en la lengua. (Arturo Azuela, La casa de las mil vírgenes (México), I 983, CREA).

(8) -En todo caso, diga lo que diga la ley, icree que políticamente el golpe es mortal para el PP y, sobre todo, para Aznar?
-Es un golpe, pero no tan mortal. Ni para Aznar ni para nadie. José María es joven, tiene mucha vida por delante, lo cual es envidiable, y merece un margen de confianza. No tiene por qué verse afectado, ni tampoco Fraga (Tiempo (España), 1990, CREA).

(9) Opina que las palabras de mi madre le parecen hermosas y replico diciéndole que tal vez puedan serlo en otras circunstancias, pero que a mí no me lo parecieron en aquel momento y que, al oírle decir todas aquellas estupideces, pensé que ya era hora de agarrar al toro por los cuernos y de hablar claramente (Javier Tomeo, Amado monstruo (España), 1985, CREA).

(I0) Como periodista de radio, como columnista, pero sobre todo desde la dirección de QAP, María Isabel se convirtió en uno de los 'cocos' de los congresistas. Más de una vez Bernardo Guerra, Alberto Santofimio, José Guerra Tulena y todos los grandes caciques electorales salieron desplumados de las emisiones de QAP. [...] Ahora, esa misma mujer que no ha tenido pelos en la lengua para señalarle al Congreso sus culpas, quiere hacer parte de él (Semana (Colombia), 1998, CREA).

(I I) Silvia: ¿Cómo me encuentras...?

Jenaro: (Enigmático) Asombrosa.

Silvia: Entonces estarás de acuerdo en que me queda mucha vida por delante y que tengo mucho que ofrecer (María Luisa Luca de Tena, Un millón por una rosa (España), 1992, CREA).

(I2) Pero el canciller no dispuso apenas de tiempo para planificar una serie de acontecimientos históricos que se sucedieron con toda rapidez, y hay que reconocer que tuvo la audacia de coger el toro por los cuernos (El Mundo (España), 1994, CREA).

(I3) El tercer triunfo de Meciar en una Eslovaquia abatida por dificultades económicas, con una tasa de desempleo que supera el $18 \%$ y la inflación alrededor del $17 \%$, se atribuye en primer lugar a sus dones populistas. Como señalaran con pesar algunos 
de sus críticos, "Meciar, con su carácter impulsivo e imagen de hombre rudo, sin pelos en la lengua para llamar a las cosas por su nombre, representa el prototipo del pensamiento eslovaco" (El Mundo (España), 1990, CREA).

Así,por un lado,en los ejemplos (4) a (8) se encuentran apoyos que glosan de manera más o menos completa el significado 'estándar' asociado a las locuciones: 'tratar de arreglar las cosas', 'enfrentarse a una situación', 'hablar con sinceridad', 'ser abierto/ franco' o 'ser joven' podrían considerarse como alternativas expresivas -estilísticamente menos marcadas- de 'agarrar/coger/entrar/tomar el/al toro por los cuernos', 'no tener pelos en la lengua/sin pelos en la lengua' $y$ 'tener/quedar a alguien (toda una/la) vida por delante'.

De otra parte, existe la posibilidad de que el apoyo refleje el modo en que el contexto referencial restringe el significado genérico codificado para el fraseologismo en cuestión. Esto se aprecia, por ejemplo, en (9), ( I0) y (I I): en los contextos citados, 'asumir una situación difícil' consiste,específicamente, en 'hablar claramente' (agarrar/coger/entrar/tomar el/al toro por los cuernos); 'decir sin miramientos lo que se piensa', en 'señalar culpas' (no tener pelos en la lengua/sin pelos en la lengua); $y$ 'ser joven', en 'tener mucho que ofrecer' (tener/quedar a alguien (toda una/la) vida por delante).

Los apoyos detectados en los testimonios (12) y (I3) -'tener audacia'; 'tener un carácter impulsivo', 'ser rudo'- contribuyen, por su parte, a reforzar semas específicos de 'agarrar/coger/entrar/tomar el/al toro por los cuernos' y 'no tener pelos en la lengua/sin pelos en la lengua', respectivamente, acotando, de nuevo, el significado idiomático de ambas expresiones.

Ahora bien, lo que interesa más en este momento es poder definir -al hilo de lo señalado respecto de los ejemplos (4)-(I3) - algunas razones que explican la frecuente aparición de los apoyos co(n)textuales. En primer lugar, si, como se ha señalado en el apartado 2. (específicamente en la sección 2.I.), el co(n) texto lingüístico de aparición de un fraseologismo suele sustentar cognitivamente su significado y su motivación figurativa, cabrá esperar que dentro de esta misma 'línea de pensamiento' puedan detectarse más expresiones -figuradas 0 no- que sigan incidiendo globalmente en el significado que queda cristalizado con la utilización de la UF. Dicho de modo más ilustrativo, en ejemplos como los citados más arriba se percibe una necesidad del hablante de glosar de modos diversos los significados 'asumir de manera decidida e inmediata un asunto difícil', 'decir sin miramientos lo que se piensa' $y$ 'ser joven $y$, por ende, tener proyectos de vida', que se expresan, tal vez, de modo cognitivamente más prominente a través de las locuciones 'agarrar/coger/entrar/ tomar el/al toro por los cuernos', 'no tener pelos en la lengua/sin pelos en la lengua' y 'tener/quedar a alguien (toda una/la) vida por delante', y cuya actualización discursiva se refuerza con estos elementos 'periféricos' que definimos como apoyos co(n)textuales.

En segundo lugar, se aprecia también que, dada la flexibilidad -0 incluso vaguedad- de su contenido semántico,ciertas UFS suelen recibir especificaciones y acotaciones en su significado, como se ha visto que sucede en el ejemplo (II): 'tener/quedar a alguien (toda una/la) vida por delante' suele suponer también, en muchos casos, 'tener mucho que ofrecer'.

Asimismo, en ocasiones el hablante parece querer aclarar expresamente el significado de la UF que inserta en su discurso, o el sentido particular en que esta debe interpretarse. Esto explicaría la coordinación o yuxtaposición -redundante, si se quiere- de los fraseologismos con sus respectivas paráfrasis no fraseológicas en casos como el presentado en (5) '(coger el toro por los cuernos/ enfrentarse a la situación)'; o, sobre todo, la adición de segmentos explicativos o expresamente reformulativos como el que se observa en este testimonio adicional de 'coger el toro por los cuernos':

( I4) Hay, pues, que "coger al toro por los cuernos", como Europa parece haberlo comprendido, ipor fin!; es decir, partir de las bases del conflicto y verlo globalmente [...] (El País (España), 1980, CREA; la cursiva es nuestra).

Podría decirse que los apoyos co(n)textuales 'juegan', en suma, con la competencia fraseológica de los hablantes, pues, aunque las UFS condensan muchas veces ideas complejas de manera efectiva y cognitivamente más accesible, suele suceder también que, dada su riqueza figurativa y su naturaleza de signos complejos, necesiten recibir apoyos como los descritos que aseguren, en cada contexto determinado, su correcta y precisa interpretación por parte del oyente. 


\section{Apoyos co(n)textuales: Tipos y aplicación}

\section{I.Tipología de los apoyos co(n)textuales}

Se presenta a continuación una tipología tentativa de las clases de apoyos co(n)textuales; clases basadas, fundamentalmente, en las características y en la relación semántica que guardan estas piezas con las UFS a las que acompañan. Con ella se persigue afinar y precisar algo más la descripción de este fenómeno discursivo y sentar las bases para seguir profundizando en su análisis en el futuro.

La tipología se ilustrará a través del corpus de testimonios fraseológicos recabado en el marco del Proyecto FRASESPAL, y que, según se indicó en el apartado 3. (ver sección 3.I. y nota 7), se compone de datos ofrecidos por el CREA, esencialmente, con apoyo de ejemplos obtenidos, asimismo, de Internet.

\section{I. I. Paráfrasis explicativas}

En primer lugar, parece que el tipo de apoyo co(n) textual más frecuente se corresponde con lo que podemos denominar 'paráfrasis explicativas' de las UFS, esto es, glosas más o menos completas del significado asociado a cada expresión.

(I5) No pienses tanto, Carmen, que te va echar humo la cabeza (<http://www.carmenposadas. net/obra-ficha.php?obra=510-08-09> 10/8/2009).

(España),

(16) Estáis buscando una explicación política a un problema que es puro cabreo social. Si el domingo pasado salieron 15.000 personas a la calle no es porque haya cuatro politiqueros que les estemos incitando, sino porque están hasta las narices de pagar tan caro el metro y el autobús, de pagar tropocientos impuestos si quieren ducharse ( $\mathrm{La}$ Vanguardia (España), I 994, CREA).

(I7) Vuelo sobre el Atlántico y dejo de quebrarme la cabeza reconstruyendo cronologías, atando cabos, resolviendo misterios (Carlos Fuentes, Constancia (México) 1997, CREA).

(I8) Nunca he separado las cosas, una vida creativa es una vida creativa, en la que puedes incluir todo el arte. Si quieres ser director de cine, tienes que saber de interpretación, de fotografía, de pintura, de música, de moda, tienes que estar al día. Para mí, es algo natural (El País (España), 2002, CREA).

(19) El Land Cruiser estaba en forma, duro, rápido, más veloz que nunca superó todos los obstáculos con alegría, como si de verdad llevara un tigre en el motor (Manuel Leguineche, El camino más corto. Una trepidante vuelta al mundo en automóvil (España), 1996, CREA).

Los ejemplos (15) a (19) reflejan, en este sentido, el continuum que parte de esta explicitación del significado genérico de la unidad -testimonio [15]: 'echarle' a alguien 'humo' la cabeza $\rightarrow$ 'pensar mucho'- y progresa hacia la inclusión de glosas cada vez más creativas, que buscan, principalmente, concretar con mucho más detalle el contenido general de la UF (sobre todo, los ejemplos (18) y (19)).

\section{I.2. Apoyo de semas relevantes del significado de las} UFS

Un tipo de apoyo co(n)textual afín, aunque no idéntico, a las paráfrasis descritas es el que focaliza particularmente alguno de lo semas que componen el significado del fraseologismo en cuestión (también ‘supra' en la sección 3.2.).

(20) Pero el canciller no dispuso apenas de tiempo para planificar una serie de acontecimientos históricos que se sucedieron con toda rapidez, y hay que reconocer que tuvo la audacia de coger el toro por los cuernos (El Mundo (España), 1994, CREA).

(2I) Hija, no le digas a tu padre que te has quedado embarazada porque del sofocón que se lleva puede darle un soponcio (Alfonso Ussía, Tratado de las buenas maneras (España), I 995, CREA).

Los ejemplos (20) -incluido ya como (12) en el apartado 3.2.- y (2I) reflejan contextos de empleo que potencian, en efecto, uno de los componentes del significado de las UFS: de un lado, la 'valentía' que caracteriza la acción denotada por 'coger el toro por los cuernos'; $y$, de otro, el 'sofocón (emocional)' que puede propiciar que a un sujeto 'le dé un soponcio'. La (leve) diferencia entre los apoyos co(n)textuales de la sección 4.I.I.y los que aquí se tratan residiría, por tanto, en que estos últimos no permiten reconstruir de manera completa el significado de la UF a la que se aplican.

\section{I.3. UFS y ULS sinónimas o semánticamente muy próximas a la UF 'central'}

(22) Es por lo tanto deseable, o al menos conveniente, que estemos al corriente sobre las últimas tendencias y cambios, ya que estos, mayoritariamente, son debidos a pequeñas innovaciones, más que a 
profundas variaciones, por lo que la puesta al día, no suele presentar mayor dificultad, y lo que es más importante daremos la imagen de estar al día y no obsoletos (Internet (España), 1999, CREA).

(23)] Pero al presidente todo esto le da igual. Le importa un pimiento el futuro de su partido (EI Mundo (España), 1995, CREA).

(24) De la noche a la mañana mi papá cambió. Dejó de tomar licor. Un día nos dijo que ya era un hombre nuevo, que había nacido de nuevo y nos pidió que fuéramos con él a su iglesia (La Hora (España), 1997, CREA).

(25) Sinceramente y con la mano en el corazón, deseo que el señor Zedillo se esfuerce por mejorar la Nación, que termine con honores su periodo presidencial (Excélsior (México), 1996, CREA).

Una nueva serie de testimonios ilustra el frecuente apoyo discursivo de las UFS en: a) otros fraseologismos 'sinónimos' (ejemplos (22) y (23)) o semánticamente 'muy próximos' a ellas (ejemplo (24)); b) unidades léxicas (ULS) también sinónimas o de significado muy próximo al suyo (ejemplo (25)) ${ }^{10}$.

\section{I.4. UFS y ULS antónimas o semánticamente contrapuestas a la UF 'central'}

De la misma manera que las UFS se coordinan discursivamente con UFS y ULS sinónimas o semánticamente afines a ellas, es posible hallar casos en los que dicha coordinación se da con elementos léxicos o fraseológicos 'antónimos' o de significado 'contrapuesto' a ellas en algún aspecto. Estas piezas apoyan, pues, la interpretación de la UF 'central' en la medida en que aparecen explícitamente 'enfrentadas' a esta, bien, por ejemplo, a través de una negación explícita (ver más abajo (26), (27)), bien mediante una coordinación disyuntiva (en (28)):

(26) Sea como sea, el caso es que la financiación irregular no se acepta, y está bien que no se acepte. De ahí que no se pueda seguir escurriendo el bulto, sino que sea necesario coger el toro por los cuernos (El País (España), I997, CREA).

(27) No cabía silenciarlo, ni hacerse el distraído.Ante un acontecimiento tan evidente había que coger el toro por los cuernos (Antonio Gala, Los invitados al jardín (España), 2002, CREA).

(28) Ramón Martínez Lao quiere saber si ante un problema pide usted ayuda o coge el toro por los cuernos (Oral (España), 1989, CREA).

(29) Inglaterra demostró, unos meses más tarde, cuando los atentados en el metro y en los autobuses de Londres, que los pueblos valientes son los que se unen y los que se ratifican ante la adversidad, no los que se van por la pata abajo, como se fueron tres millones de españoles que son los que cambian la línea de quien gana y quien pierde. (<http:// zaragozaunica.com/2007/0 I/26/el-nombre-del-diazapatero-salio-por-la-cobardia-del-pueblo-espanol> (España), 2/8/2009).

\section{I.5. Otras consideraciones}

No existen, por supuesto, impedimentos para que varios de estos tipos de apoyos contribuyan conjuntamente al soporte conceptual del empleo discursivo de los fraseologismos. Esto se aprecia en ejemplos como el que sigue, donde la locución 'de primera mano' se combina con otra UF semánticamente muy afín a ella (de buena tinta) y se ve glosada, además, por una paráfrasis explicativa (nada de rumores infundados ni tonterías derrotistas):

(30) Doña Ramona siempre decía que las informaciones que ella tenía eran "de primera mano", "de buena tinta". Es decir, nada de rumores infundados ni tonterías derrotistas. Ella sabía con quién trataba (Inés Palou, Carne apaleada (España), 1975, CREA).

\subsection{Alcance del empleo de los apoyos co(n) textuales}

Los testimonios citados hasta el momento demuestran que la inserción de los apoyos co(n) textuales impregna el uso real de 'todo tipo' de UFS. Por un lado, estos apoyos interpretativos pueden acompañar a expresiones 'descriptivas' que presentan grados 'diversos de idiomaticidad': desde la alta transparencia semántica de '[ser] (un) hombre nuevo' (en el ejemplo (24)) o 'coger el toro por los cuernos', integrante del análisis cuantitativo de la sección 3., hasta la opacidad figurativa de 'dorar la píldora', por ejemplo:

(31) Andrés Manuel López Obrador advierte que el presidente Ernesto Zedillo y el secretario de Gobernación, Emilio Chuayffet, "saben que yo no me dejo dorar la píldora. Nosotros no vamos a permitir engaños" (Proceso (México), I 996, CREA).

En efecto, según estos datos, podría decirse que es el significado fraseológico ya fijado y conocido por los hablantes el que recibe los apoyos co(n)textuales, 
con independencia de que dichos hablantes puedan acceder realmente a la motivación metafórica o metonímica de la UF en cuestión.

De otra parte, también los fraseologismos de 'valor pragmático' pueden recibir este tipo de aclaraciones acerca de la función discursiva que desempeñan en el contexto particular de su enunciación. En otras palabras, su 'significado pragmático' (más arriba en la sección 2.2., especialmente la nota 6) también se ve reforzado en ocasiones por apoyos co(n)textuales de variada naturaleza:

(32) Sinceramente y con la mano en el corazón, deseo que el señor Zedillo se esfuerce por mejorar la Nación, que termine con honores su periodo presidencial (Excélsior (México), I 996, CREA).

(33) Si hay algo que a los Magos de Oriente no les gusta es la gente malhablada así que a la antigua usanza... lávate la boca con jabón y que no salga ni una sola palabra malsonante de ese orificio (<http:// events.es.msn.com/navidad/entretenimiento/ fotos. aspx?cpdocumentid $=|50850|$ 23\&imageindex $=7>$ (España), 0I/20I0).

En efecto, en (32) -citado como (25) en el apartado 4.I.3. -, se observa la coordinación de la locución modal 'con la mano en el corazón' con su equivalente en la esfera de los adverbios modalizadores '(sinceramente)'. Por otra parte, en (33) es una fórmula rutinaria de valor expresivodirectivo -'lávate la boca con jabón', con la que un hablante transmite una queja acerca del mal hablar de su interlocutor y le invita, en consecuencia, a expresarse de manera adecuada (Olza, 2009) - la que recibe el apoyo interpretativo de la paráfrasis -también directiva- 'que no salga ni una sola palabra malsonante de ese orificio'.

\section{CONCLUSIONES}

Consideramos que los indicios presentados en este trabajo sugieren sendas interesantes de análisis de la aparición de los apoyos co(n)textuales como una tendencia significativa del empleo discursivo de las UFS. Ahora bien, somos conscientes de que el conjunto de mecanismos que hemos englobado bajo el marbete de 'apoyo co(n)textual' reclama todavía un examen ciertamente más extenso y riguroso que el presentado aquí, dada su evidente heterogeneidad y las fronteras difusas que presenta con otros fenómenos destacados en el uso discursivo de la fraseología". En este sentido, no podemos sustraernos al hecho de que el análisis de la fraseología 'en contexto' resulta, cuando menos, difícil de sistematizar, dada, también, la (relativa) escasez de herramientas metodológicas desarrolladas hasta el momento con este objetivo.

Con todo, cabe cerrar este estudio ponderando las interesantes aplicaciones ulteriores del análisis de los fenómenos de apoyo discursivo de las UFS, pues su estudio puede ayudar, entre otros aspectos, a seguir profundizando en la comprensión de los procesos cognitivos que subyacen al empleo de la fraseología (aplicaciones en el terreno de la psicolingüística); o, en otro orden de cosas, a afinar, dentro de la práctica lexicográfica, en la definición y ejemplificación de los significados fraseológicos, en la medida en que los testimonios citados en este trabajo podrían tener cabida como muestras verdaderamente 'explicativas' $-y$ no meramente documentativas- del valor semántico-pragmático real de las UFS (Mellado Blanco, 2009) ${ }^{12}$. 


\section{REFERENCIAS BIBLIOGRÁFICAS}

Alvarado Ortega, Ma B. (2008). Las fórmulas rutinarias en el español actual. Tesis doctoral, Universidad de Alicante, Alicante, España.

Casado, M. (1993). Introducción a la gramática del texto del español. Madrid:Arco/Libros.

Corpas Pastor, G. (1996). Manual de fraseología española. Madrid: Gredos.

Gibbs, R.W. (1993). Why idioms are not dead metaphors. En C. Cacciari \& P. Tabossi (Eds.), Idioms: Processing, structure, and interpretation (pp. 57-77). Hillsdale: Erlbaum.

Gibbs, R.W. (1994). The poetics of mind. Figurative thought, language, and understanding. Cambridge: Cambridge University Press.

Giráldez Soage, A. (2006). El papel de las cercas semánticas (hedges) en las estrategias comunicativas discursivas. En M. Casado, R. González \& M.V. Romero (Eds.), Análisis del discurso: Lengua, cultura, valores. Actas del I Congreso Internacional (pp. 475-488). Madrid: Arco/Libros.

Mellado Blanco, C. (2009). Utilidad y limitaciones de los corpora informáticos en la elaboración de un tesauro fraseológico (alemán-español). En P. Cantos \& A. Sánchez (Eds.), A survey on corpus-based research/Panorama de investigaciones basadas en corpus (pp. I38-I5I). Murcia:Asociación Española de Lingüística de Corpus.

Montoro del Arco, E. T. (2006). Teoría fraseológica de las locuciones particulares. Las locuciones prepositivas, conjuntivas y marcadoras en español. Frankfurt am Main: Peter Lang.

Olímpio de Oliveira Silva, Ma .E.(2006). Reflexiones sobre el uso de los marcadores fraseológicos. En M. Casado, R. González \& Mª. V. Romero (Eds.), Análisis del discurso: Lengua, cultura, valores. Actas del I Congreso Internacional (pp. I5 I3-1524). Madrid:Arco/Libros.

Olza, I. (2009). Aspectos de la semántica de las unidades fraseológicas. La fraseología somática metalingüística del español. Tesis doctoral, Universidad de Navarra, Pamplona, España.

Olza,I.(2010).Presentadores fraseológicos y actitudes de enunciación en la argumentación periodística. Presentado en el $9^{\circ}$ Congreso Internacional de Lingüística General, Universidad de Valladolid, España.

Penadés Martínez, I. (2000). La hiponimia en las unidades fraseológicas. Cádiz: Universidad.

Rastier, F. (I 976). Sistemática de las isotopías. En A.J. Greimas (Dir.), Ensayos de semiótica poética (pp. I07-I40). Barcelona: Planeta.

Real Academia Española (2008). CREA. Corpus de Referencia del Español Actual. Real Academia Española [en línea]. Disponible en: http://corpus.rae.es/creanet.html

Ruiz, L. (2005). Las locuciones marcadoras del español:Análisis y aplicaciones. En R. Almela, E. Ramón Trives \& G.Wotjak (Eds.), Fraseología contrastiva: Con ejemplos tomados del alemán, español, francés e italiano (pp. 24I-257). Murcia: Universidad.

Sancho Cremades, P. (1999). Introducció a la fraseologia. Aplicació al valencià col.loquial. Paiporta: Editoria Denes.

van Dijk, T. (1972). Some aspects of text grammars.A study in theoretical linguistics and poetics. The Hague/Paris: Mouton. 


\section{NOTAS}

I La realización de este trabajo se enmarca en el Proyecto de Investigación 'La estructura idiomática del alemán y el español. Un estudio cognitivo a partir de un corpus onomasiológico' (Ministerio de Ciencia e Innovación; código HUM2007-62198/ FILO), desarrollado por el grupo FRASESPAL (www. usc.es/ frasespal); e, igualmente, en los Proyectos de Investigación 'El discurso público: Estrategias persuasivas y de interpretación', desarrollado por GRADUN (Grupo Análisis del Discurso. Universidad de Navarra; (www.unav.es/centro/ analisisdeldiscurso) en el seno del ICS (Instituto Cultura y Sociedad) de la Universidad de Navarra, y 'Metodología de análisis del discurso: Propuesta de una lingüística del texto integral' (Ministerio de Ciencia e Innovación; código FFI20I0-204l6). Algunos aspectos de este estudio se presentaron en el XXXIX Simposio Internacional de la Sociedad Española de Lingüística (Universidad de Santiago de Compostela, I-4 febrero, 20I0).

2 Para la investigación en español sobre las fórmulas rutinarias, basta con citar las líneas de análisis sentadas tempranamente por Corpas Pastor (1996), que han recibido el relevo de aportaciones monográficas como la de Alvarado Ortega (2008). Las locuciones marcadoras han sido tratadas y definidas en profundidad por Ruiz (2005) y por Montoro del Arco (2006), entre otros.

3 Esto no impide, sin embargo, que las funciones - cognitivo-discursivas (ver apartado 2.)desempeñadas por los apoyos co(n)textuales puedan relacionarse, por ejemplo, con las labores de presentación y marcación que llevan a cabo los denominados 'marcadores', 'conectores' o 'presentadores fraseológicos', que sí se han prestado, en cambio, a ser analizados como una categoría funcional de piezas de valor pragmático-discursivo (más abajo sección 2.2.; Sancho Cremades (I999); Olímpio de Oliveira Silva (2006); Mellado Blanco (2009); Olza (20I0)).

$4 \mathrm{Si}$ bien el estudio de estos marcadores o presentadores se ha centrado más en su frecuencia de coaparición con las 'paremias' -ver la mención de los 'presentadores paremiológicos' realizada, por ejemplo, por Corpas Pastor (1996)-, actualmente se ha ampliado a su aplicación a todo tipo de UFS, que también son 'presentadas' en su calidad de segmentos de discurso repetido diatópica, diastrática y diafásicamente marcados (más abajo).
De hecho, el testimonio (3), incluido más abajo, ilustra la combinación del marcador fraseológico 'como vulgarmente se dice' con una 'locución (coger el toro por los cuernos)'.

5 Otras funciones de estos marcadores fraseológicos -por ejemplo, la atenuación de la responsabilidad enunciativa del emisor- han sido descritas por Sancho Cremades (1999), Olímpio de Oliveira Silva (2006), Mellado Blanco (2009) u Olza (20I0). Consideramos, no obstante (Olza, 20I0), que este tipo de piezas reclaman aún una atención profunda en el marco del estudio de la fraseología en contexto, y que, de hecho, podrían encuadrarse dentro del análisis de otros mecanismos discursivos de mayor alcance, como el empleo de hedges o 'cercas discursivas' (piezas muy variadas de función semántica y pragmáticamente matizadora respecto del segmento que introducen:'así llamado','hablando entre nosotros', 'especie de', etc. (Giráldez Soage, 2006).

6 En la sección 4. (apartado 4.2.) se verá cómo esta incidencia en el nivel del significado de las UFS afecta al 'contenido semántico-proposicional' que poseen las unidades de tipo descriptivo, pero también al 'significado pragmático-discursivo' que presentan ciertas UFS.

7 Los ejemplos del corpus FRASESPAL para los fraseologismos del español se toman del CREA, fundamentalmente, y también, aunque en menor medida, de Internet. En esta sección prestaremos atención a los testimonios del CREA como corpus representativo del español actual, pero en la sección 4. también se incluirán ejemplos del corpus FRASESPAL obtenidos de Internet.

\section{Fecha de la consulta: 7/10/2010.}

9 Nótese que el volumen de testimonios arrojados por el CREA para las tres expresiones es relativamente similar.

10 Esta confluencia sintagmática de UFS y ULS sinónimas -y antónimas (más abajo sección 4.I.4.)demuestra la conveniencia de analizar la fraseología y el léxico desde la óptica de las relaciones semánticas estructurales -sinonimia, antonimia, hiperonimia, hiponimia- que adquieren, efectivamente, en cada lengua (Penadés Martínez, 2000; Olza, 2009). 
I I Nos referemos aquí, por ejemplo, al empleo de los ya mencionados 'marcadores' o 'presentadores fraseológicos' (sección 2.2.); a los variados 'comentarios metalingüísticos' que a veces guían 'reflexivamente' la interpretación de las UFS (más abajo en (34)); o a los juegos de 'desautomatización' a que se ven sujetos con llamativa frecuencia los fraseologismos (35):

(34) ¡Bocachancla! ¡Es usted un bocachancla!. Qué plástica expresión. Qué rotundo remate de discusión de parlamentarios. Eso fue lo que le dijo el otro día el Sr. Aguirre (PNV) al Sr. Urquijo (PP) después de que éste dijera de aquél que es un recaudador de impuestos revolucionarios (<http:// elpuente.blogia.com/2006/ 06250I-bocachancla. php> (España), 25/6/2006).
(35) Me gustó cómo se refirió al Ejército Zapatista. "Por otro lado, había momentos en el Informe de Zedillo que parecía que se estaba refiriendo a otro país que no era el nuestro. A uno idílico. Más que dorarnos la píldora, nos la estaba enrosando, ya que a fuerzas nos quería presentar una realidad pintada de color de rosa. "Yo creo que no lee los periódicos", me dije en un momento dado (Diario de Yucatán (México), 1996, CREA).

I2 A este tipo de ejemplificación aspira, de hecho, la construcción del tesauro fraseológico alemánespañol que se está llevando a cabo en el seno del Proyecto FRASESPAL (ver sección 3.I. y notas I y 7). 